\title{
Multifrequency Observations of the Virgo Blazars 3C 273 and 3C 279 in CGRO Cycle 8
}

\author{
W. Collmar ${ }^{1}$, S. Benlloch ${ }^{2}$, J.E. Grove ${ }^{3}$, R.C. Hartman ${ }^{4}$, \\ W.A. Heindl ${ }^{5}$, A. Kraus ${ }^{6}$, H. Teräsranta ${ }^{7}$, M. Villata ${ }^{8}$, K. Bennett ${ }^{9}$, \\ H. Bloemen ${ }^{10}$, W.N. Johnson ${ }^{3}$, T.P. Krichbaum ${ }^{6}$, C.M. Raiteri ${ }^{8}$, \\ J. Ryan ${ }^{11}$, G. Sobrito ${ }^{8}$, V. Schönfelder ${ }^{1}$, O.R. Williams ${ }^{9}$, J. Wilms ${ }^{2}$ \\ ${ }^{1}$ Max-Planck-Institut für extraterrestrische Physik, Postfach1603, 85740 Garching, Germany \\ ${ }^{2}$ Institut für Astronomie und Astrophysik, Univ. of Tübingen, Tübingen, Germany \\ ${ }^{3}$ Naval Research Lab., 4555 Overlook Av., SW, Washington, DC 20375-5352, USA \\ ${ }^{4}$ NASA/Goddard Space Flight Center, Greenbelt, MD 20771 \\ ${ }^{5}$ Center for Astrophysics and Space Sciences, UCSD, La Jolla, CA, USA \\ ${ }^{6}$ Max-Planck-Institut für Radioastronomie, D-53121 Bonn, Germany \\ ${ }^{7}$ Metsähovi Radio Research Station, FIN-02540 Kylmälä, Finland \\ ${ }^{8}$ Osservatorio Astronomico di Torino, I-10025 Pino Torinese, Italy \\ ${ }^{9}$ Astrophysics Division, ESTEC, NL-2200 AG Noordwijk, The Netherlands \\ ${ }^{10}$ SRON-Utrecht, Sorbonnelaan 2, NL-3584 CA Utrecht, The Netherlands \\ ${ }^{11}$ Universtity of New Hampshire, Durham NH 03824-3525, USA
}

\begin{abstract}
We report first observational results of multifrequency campaigns on the prominent Virgo blazars 3C 273 and 3C 279 which were carried out in January and February 1999. Both blazars are detected from radio to $\gamma$-ray energies. We present the measured X- to $\gamma$-ray spectra of both sources, and for 3C 279 we compare the 1999 broad-band (radio to $\gamma$-ray) spectrum to measured previous ones.
\end{abstract}

\section{INTRODUCTION}

We report on simultaneous multifrequency observations of the prominent Virgo blazars 3C 273 and 3C 279 during CGRO Cycle 8. Because both blazars are known $\gamma$-ray sources, which have been detected by the CGRO experiments several times before, we proposed for simultaneous CGRO (OSSE, COMPTEL) and RXTE high-energy observations. The prime goal was to simultaneously measure their highenergy spectra from about $2.5 \mathrm{keV}$ to $30 \mathrm{MeV}$. Because of the shortage of spark chamber gas, the EGRET experiment is hardly available anymore and therefore was not requested in the proposals. After the proposed simultaneous high-energy observations were approved and scheduled, additional simultaneous observations 
TABLE 1. Summary of the satellite observations of both Virgo blazars during the campaigns in 1999. The observation periods as well as the coverage in energy are given.

\begin{tabular}{lccc}
\hline Source & Experiment & Obs. Period in '99 & Energy Band \\
\hline 3C 273 & EGRET & Jan. 20 - Feb. 2 & $30 \mathrm{MeV}-10 \mathrm{GeV}$ \\
& COMPTEL & Jan. 5 - Feb. 2 & $750 \mathrm{keV}-30 \mathrm{MeV}$ \\
& OSSE & Jan. 19 - Jan. 26 & $50 \mathrm{keV}-\sim 1 \mathrm{MeV}$ \\
& RXTE $^{\text {a }}$ & Jan 19, 26, Feb. 1 & $2.5 \mathrm{keV}-\sim 200 \mathrm{keV}$ \\
\hline 3C 279 $^{\text {EGRET }}$ & Jan. 20 - Feb. 2 & $30 \mathrm{MeV}-10 \mathrm{GeV}$ \\
& COMPTEL $^{\text {OOn. 5 - Feb. 2 }}$ & $750 \mathrm{keV}-30 \mathrm{MeV}$ \\
& OSSE & Jan. 5 - Jan. 19 & $50 \mathrm{keV}-\sim 1 \mathrm{MeV}$ \\
& OSSE $^{b}$ & Jan. 26 - Feb. 2 & $50 \mathrm{keV} \mathrm{-} \sim 1 \mathrm{MeV}$ \\
RXTE $^{a}$ & Jan 5, 12, 17 & $2.5 \mathrm{keV} \mathrm{-} 200 \mathrm{keV}$ \\
\hline
\end{tabular}

a 3 individual pointings of $\sim 13 \mathrm{ksec}$ each

b 3C 279 ToO observation; not reported here

were performed from ground-based observers extending the energy range of the campaigns to lower energies.

In this paper we report first observational results of the campaigns with emphasis on the $\mathrm{X}$ - and $\gamma$-ray part. In particular we present the measured $\mathrm{X}$ - to $\gamma$-ray spectra of both sources.

\section{OBSERVATIONS}

The multifrequency observations were carried out between 1999 January 5 and February 2. The observational strategy was that both blazars are within the COMPTEL field-of-view for the whole 4 weeks reaching the optimal sensitivity for the $\gamma$-ray observations, and OSSE observes simultaneously each source for 2 weeks. Within these two-week OSSE periods, three RXTE pointings were scheduled for each source covering simultaneously the X-and hard X-ray part of the spectrum and providing information on the X-ray variability. To supplement these high-energy observations both sources were simultaneously observed in different optical and radio bands. On 1999 January 15 the optical flux of 3C 279 reached a high level which - after some discussion - triggered target-of-opportunity (ToO) observations of this source. This resulted in a switch-on of EGRET which led to EGRET observations of both quasars during roughly the second half of the campaigns. Because of the 3C 279 ToO, the OSSE 3C 273 observation was stopped after one week, and OSSE observed 3C 279 again. These EGRET and OSSE ToO observations of 3C 279 are property of a different CGRO proposal and therefore their results are not reported here. The detailed high-energy observational log is given in Table 1. 


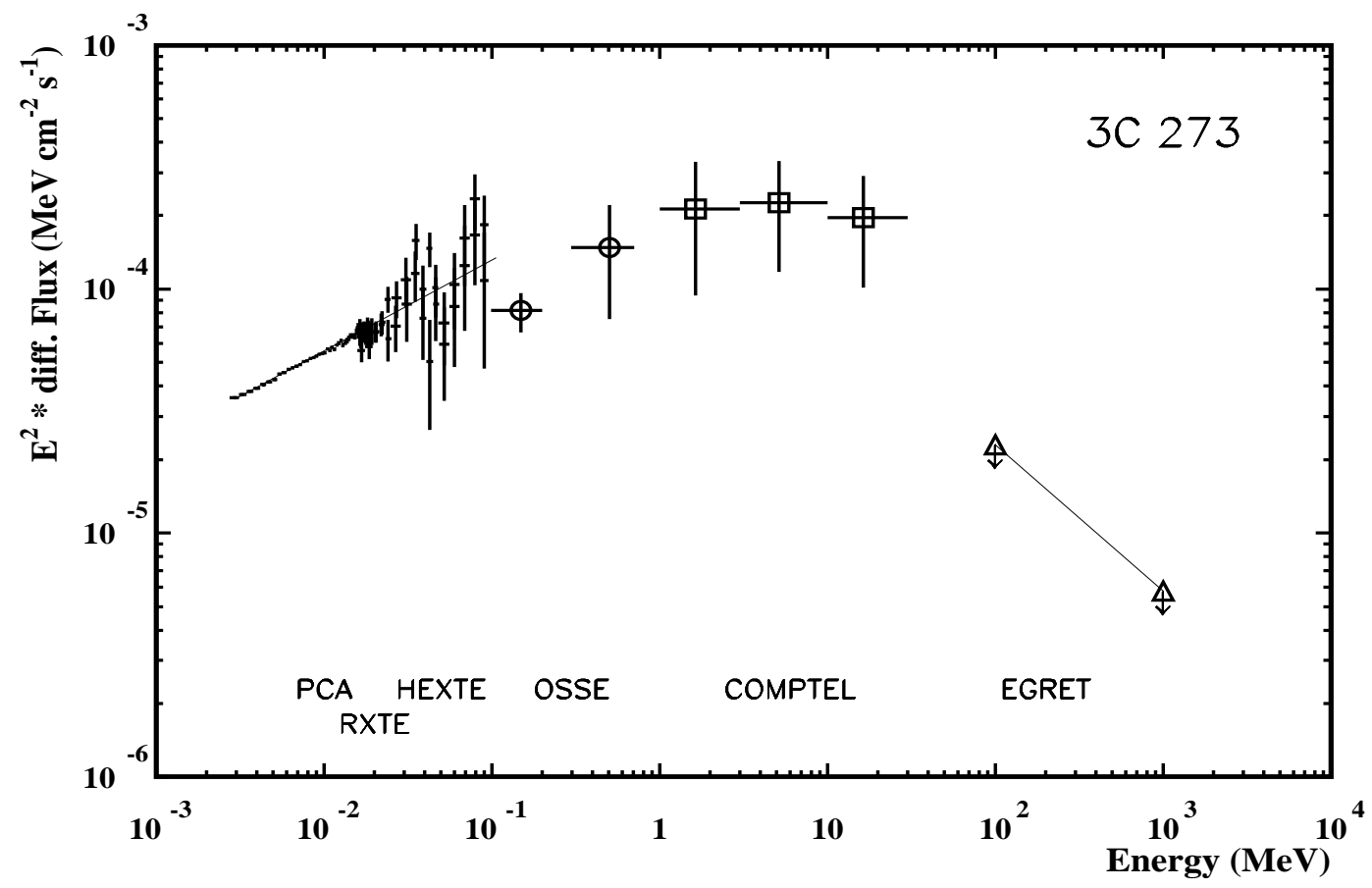

FIGURE 1. Simultaneous X-ray and $\gamma$-ray spectrum of 3C 273 as observed during the January/February 1999 campaign. The data points from the different experiments are derived from their total observation times during the campaign, which are given in Table 1 . The RXTE $(+)$ spectral points are derived from the observation sum of the three individual pointings, and are shown together with the best-fit power-law spectrum (solid line). EGRET did not detect the quasar at energies above $100 \mathrm{MeV}$ providing an upper limit on the flux. For the upper limit line, drawn between $100 \mathrm{MeV}$ and $1 \mathrm{GeV}$, a spectral power-law shape with photon index of 2.6 is assumed. The error bars are $1 \sigma$.

\section{RESULTS}

\section{$3 \mathrm{C} 273$}

3C 273 is significantly detected in all observed low-energy (radio, optical) bands. At high energies the quasar is significantly detected in $\mathrm{X}$ - and hard X-rays by RXTE from about 2.5 to $\sim 100 \mathrm{keV}$, showing a power-law spectrum with a photon index $\left(\mathrm{E}^{-\alpha}\right) \alpha$ of 1.6. OSSE detects the source in the one-week observation at a significance level of $\sim 5 \sigma$. Therefore its spectrum had to be rebinned severely to reach two significant spectral points. COMPTEL detects the blazar in the sum of the 4 -week observation at the $\sim 5 \sigma$ level. However, despite the COMPTEL detection at MeV-energies, EGRET - covering only half of the COMPTEL observation time - does not detect 3C 273 at energies above $100 \mathrm{MeV}$. The combined - more or less simultaneous - high-energy spectrum of $3 \mathrm{C} 273$ is shown in Figure 1. The 


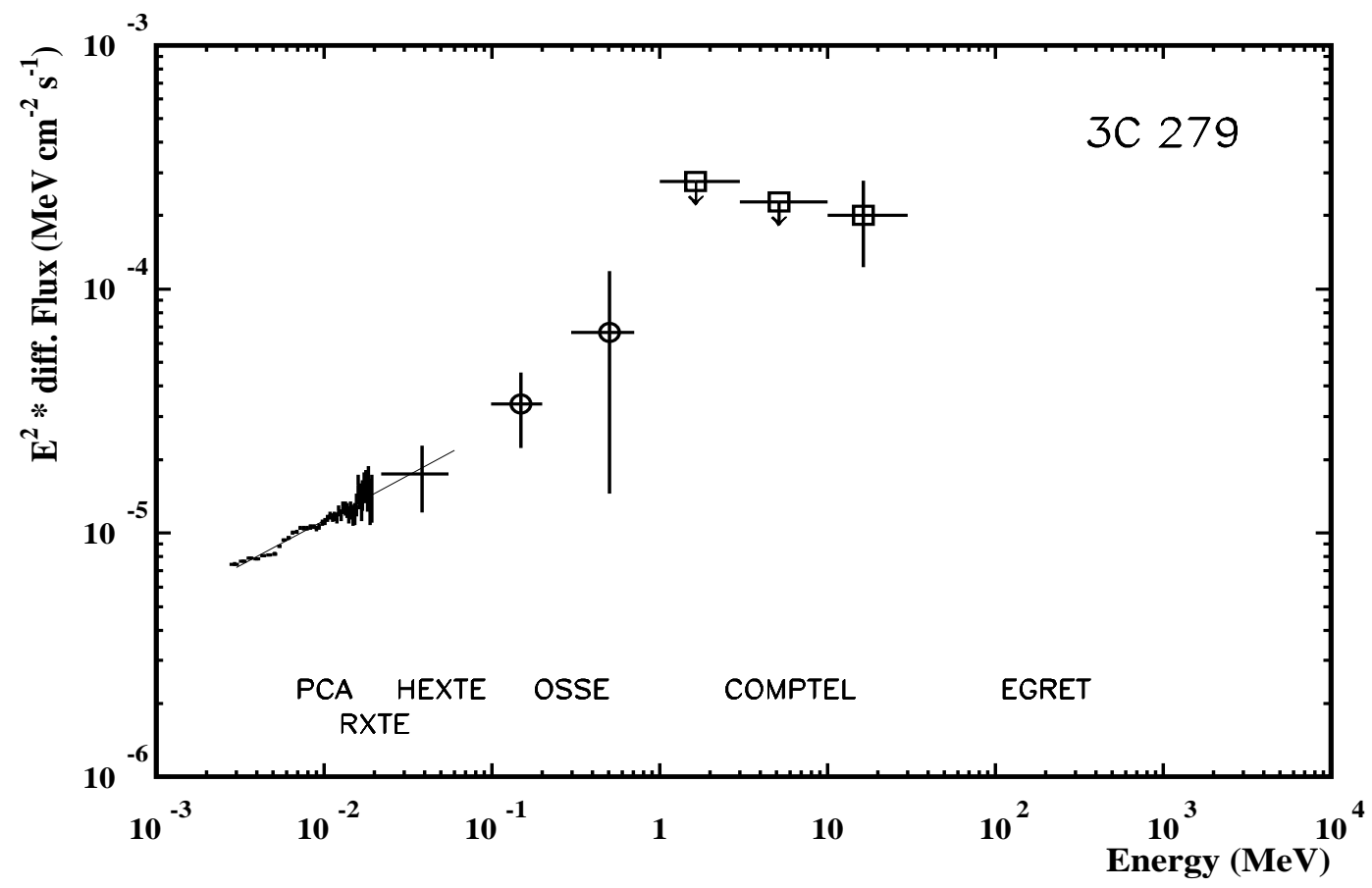

FIGURE 2. Quasi-simultaneous keV- to MeV-spectrum of the $\gamma$-ray blazar 3C 279 as observed in early 1999. The data points from the different experiments are derived from their total observations times during the campaign, which are given in Table 1 . The RXTE $(+)$ spectral points are derived from the observation sum of the three individual pointings, and are shown together with the best-fit power-law spectrum (solid line). The error bars are $1 \sigma$ and the upper limits are $2 \sigma$. Simultaneous EGRET observations exist (Table 1), which will be reported elsewhere. The spectrum can be described by a single power-law shape from about $2.5 \mathrm{keV}$ to $30 \mathrm{MeV}$.

well-known bending (e.g. [1], [2]) at MeV-energies is visible. The most surprising result however, is the non-detection by EGRET at high-energy $\gamma$-rays, despite the COMPTEL detection at $\mathrm{MeV}$-energies. This requires a strong spectral turnover between 30 and $100 \mathrm{MeV}$, and might hint at different generation mechanisms for the $\mathrm{MeV}$ and $>100 \mathrm{MeV}$ photon populations.

\section{$3 \mathrm{C} 279$}

3C 279 is significantly detected at the radio and optical bands, showing strong time variability and flaring activity in the optical. At X-rays the blazar is significantly observed up to $20 \mathrm{keV}$ by the RXTE/PCA, and is detected in hard X-rays between 20 and $50 \mathrm{keV}$ by RXTE/HEXTE with a significance of about $5 \sigma$. The RXTE/PCA spectrum is well fitted by a single power-law model with an index $\alpha$ of 1.6. At higher energies the detection significances become marginal. OSSE 


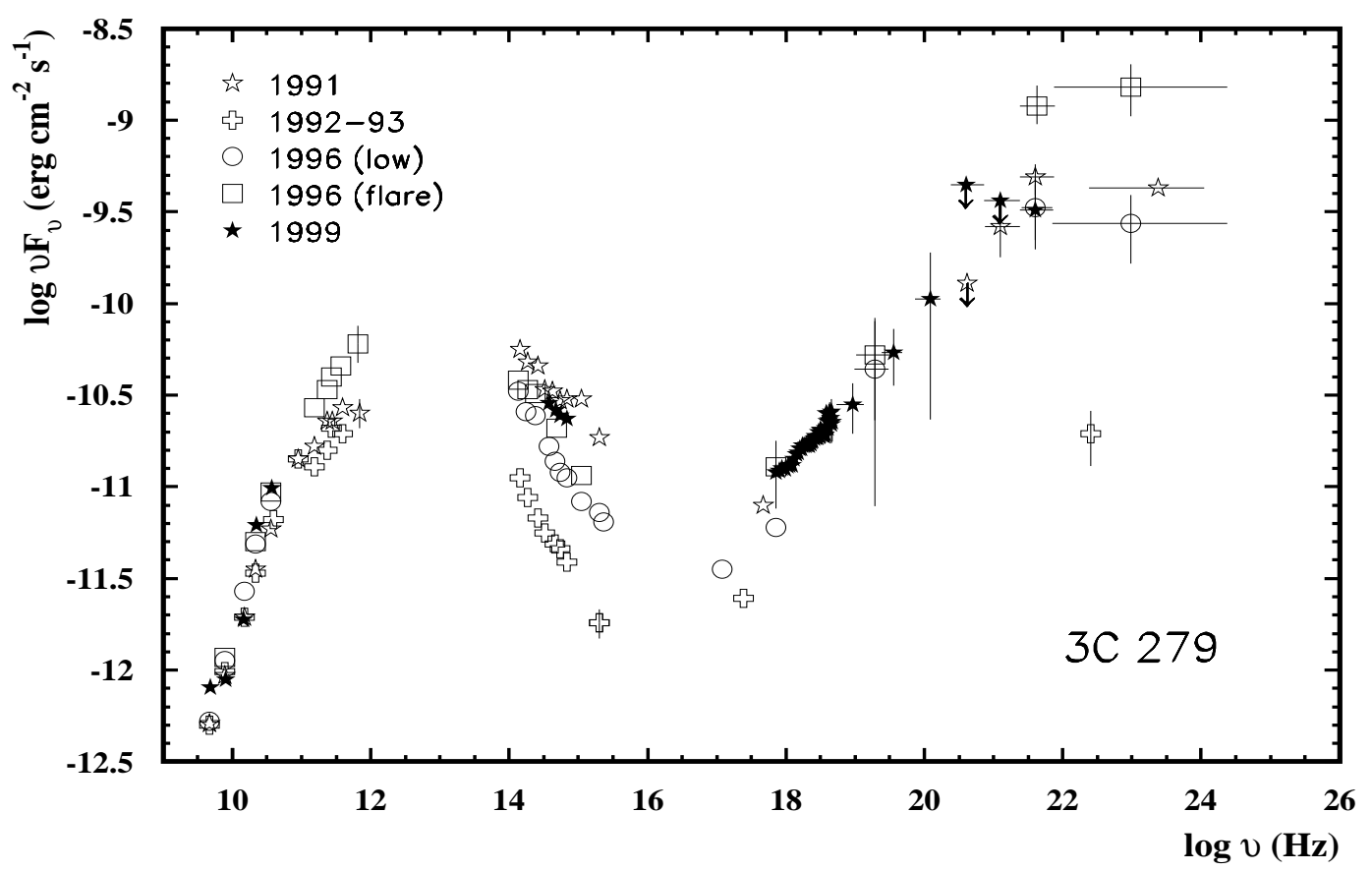

FIGURE 3. Broad-band spectrum of 3C 279 for different epochs. The 1999 results (filled stars) reported here fit nicely to the two previous measurements in 1991 and 1996 when 3C 279 was in a $\gamma$-ray high state. The spectral points of the earlier measurements are taken from [4].

found $3 \sigma$-evidence for the source only at their lower energies (near $100 \mathrm{keV}$ ), and COMPTEL - also at the $3 \sigma$ level - only at their upper energies. At energies above $100 \mathrm{MeV}$ EGRET has significantly detected 3C 279 ([3]), however these data are not reported here. The measured X- to $\gamma$-ray spectrum of 3C 279 is given in Figure 2. It shows that $3 \mathrm{C} 279$ was observed in a bright $\gamma$-ray state. The flux at the COMPTEL upper energies is at the same level as measured during the two previous $\gamma$-ray high states in 1991 and 1996 (Figure 3). The spectral power-law shape measured from $\sim 2.5$ to $20 \mathrm{keV}$ can - according to the current state of analysis - be extrapolated up to $30 \mathrm{MeV}$ without any obvious breaks or bendings. This suggests that this part of the spectrum, which is considered to be non-thermal inverse-Compton radiation, is emitted by a single emission component or mechanism.

\section{REFERENCES}

1. Lichti, G.G., et al., $A \mathscr{E} A$ 298, 711 (1995).

2. v. Montigny, C., et al., ApJ 483, 161 (1997).

3. Hartman, R.C., priv. comm., (1999).

4. Wehrle, A.E., et al., ApJ 497, 178 (1998). 